\title{
Imaging of cAMP Levels and Protein Kinase A Activity Reveals That Retinal Waves Drive Oscillations in Second-Messenger Cascades
}

\author{
Timothy A. Dunn, ${ }^{1 \star}$ Chih-Tien Wang, ${ }^{1 *}$ Michael A. Colicos, ${ }^{1}$ Manuela Zaccolo, ${ }^{3}$ Lisa M. DiPilato, ${ }^{4,5}$ Jin Zhang, ${ }^{2,4,5}$ \\ Roger Y. Tsien, ${ }^{2}$ and Marla B. Feller ${ }^{1}$ \\ ${ }^{1}$ Neurobiology Section, Division of Biological Sciences, and ${ }^{2}$ Departments of Pharmacology and Chemistry/Biochemistry and Howard Hughes Medical \\ Institute, University of California, San Diego, La Jolla, California 92093, and ${ }^{3}$ Dulbecco Telethon Institute, Venetian Institute of Molecular Medicine, I- \\ 35129 Padua, Italy, and Departments of ${ }^{4}$ Pharmacology and ${ }^{5}$ Molecular Sciences and Neuroscience, The Johns Hopkins University School of Medicine, \\ Baltimore, Maryland 21205
}

\begin{abstract}
Recent evidence demonstrates that low-frequency oscillations of intracellular calcium on timescales of seconds to minutes drive distinct aspects of neuronal development, but the mechanisms by which these calcium transients are coupled to signaling cascades are not well understood. Here we test the hypothesis that spontaneous electrical activity activates protein kinase A (PKA). We use live-cell indicators to observe spontaneous and evoked changes in cAMP levels and PKA activity in developing retinal neurons. Expression of cAMP and PKA indicators in neonatal rat retinal explants reveals spontaneous oscillations in PKA activity that are temporally correlated with spontaneous depolarizations associated with retinal waves. In response to short applications of forskolin, dopamine, or high-potassium concentration, we image an increase in cAMP levels and PKA activity, indicating that this second-messenger pathway can be activated quickly by neural activity. Depolarization-evoked increases in PKA activity were blocked by the removal of extracellular calcium, indicating that they are mediated by a calcium-dependent mechanism. These findings demonstrate for the first time that spontaneous activity in developing circuits is correlated with activation of the cAMP/PKA pathway and that PKA activity is turned on and off on the timescale of tens of seconds. These results show a link between neural activity and an intracellular biochemical cascade associated with plasticity, axon guidance, and neural differentiation.
\end{abstract}

Key words: ICUE; activity-dependent development; AKAR; cAMP-dependent protein kinase; retinal ganglion cell; spontaneous activity

\section{Introduction}

Neuronal activity is linked to long-term changes in cells, such as activity-dependent expression of genes, by activation of secondmessenger cascades (West et al., 2002). Although several such cascades have been identified, there is a short list of second messengers, such as calcium, cAMP, cGMP, DAG, and IP3, that mediate these effects. Therefore, the activation of specific cascades is not likely to be determined by overall concentrations of second messengers but rather by subcellular spatial localization and dynamic changes in their concentrations. Oscillations have been detected in both calcium and CAMP concentrations, and there is

Received April 25, 2006; revised 0ct. 27, 2006; accepted 0ct. 28, 2006.

This work was supported by National Institutes of Health Grants EY13528 and EY016417 (M.B.F.), NS27177 (R.Y.T.), and DK73368 (J.Z.), the McKnight Scholars Fund (M.B.F.), Telethon Italy Grants TCP00089 and GGP05113, the Fondazione Compagnia di San Paolo, and Human Frontier Science Program Organization Grant RGP0001/2005-C (M.Z.). L.M.D. was supported by National Research Service Award F31 DK074381 from National Institutes of Health We thank Nick Spitzer and Aaron Blankenship for helpful comments on this manuscript.

*T.A.D. and C.-T.W. contributed equally to this work.

Correspondence should be addressed to Marla B. Feller, Neurobiology Section 0357, University of California, San Diego, 9500 Gilman Drive, La Jolla, CA 92093-0357. E-mail: mfeller@ucsd.edu.

M. A. Colicos's present address: Department of Physiology and Biophysics, University of Calgary, Calgary, Alberta, Canada T2N 4N1.

DOI:10.1523/JNEUROSCI.3238-06.2006

Copyright $\odot 2006$ Society for Neuroscience $\quad$ 0270-6474/06/2612807-09\$15.00/0 growing evidence that these oscillations are "tuned" to drive particular cellular events (Zaccolo and Pozzan, 2003; Spitzer et al., 2004; Fields et al., 2005; Dyachok et al., 2006).

The establishment and refinement of retinal projection patterns occurs at an early stage of development, before the development of vision (for review, see Grubb and Thompson, 2004; Ruthazer and Cline, 2004; Torborg and Feller, 2005). During this time, retinal ganglion cells (RGCs) exhibit spontaneous bursts of action potentials that periodically propagate across the developing ganglion cell layer, an activity pattern termed retinal waves (Meister et al., 1991; Wong et al., 1993). The cAMP/protein kinase A (PKA) pathway has been implicated in the establishment and normal refinement of RGC axonal projections. Knock-out mice lacking calcium-activated adenylate cyclase 1, an enzyme that produces cAMP, exhibit defects in retinotopic and eyespecific refinement (Ravary et al., 2003; Plas et al., 2004). Knockdown of the transcription factor cAMP response elementbinding protein, a known target of PKA, leads to a reduction in eye-specific segregation (Pham et al., 2001). These effects of the cAMP/PKA pathway on refinement of retinal projections may be mediated by activation of this pathway in RGCs, perhaps by regulating the response of RGC growth cones to guidance molecules (Nicol et al., 2006) or modulating release probability at RGC 
axon terminals (Lu et al., 2006). Although spontaneous patterned activity and the cAMP/PKA pathway are important for the establishment of retinal projections, how retinal waves affect the cAMP/PKA second-messenger cascades either in RGCs or their targets remains unknown.

Here we used three genetically encoded fluorescence-based indicators to monitor the cAMP/PKA pathway in both dissociated retinal neurons and the intact retina. The indicators are as follows: (1) a PKA-based cAMP sensor (CS) (Zaccolo et al., 2002), (2) ICUE2 (indicator of cAMP using Epac), which is modified from ICUE1 (DiPilato et al., 2004), and (3) A-kinase activity reporter AKAR2.2 (Zhang et al., 2001, 2005), which undergoes changes in its fluorescent properties in response to phosphorylation by PKA. To demonstrate the utility of CS, ICUE2, and AKAR2.2, we compared their responses to direct activation of adenylate cyclase with activation of G-protein-coupled receptors by dopamine and to depolarization. We also detected spontaneous oscillations in cAMP levels and PKA activity in retinal explants. These oscillations were blocked by agents that block retinal waves and exhibited a temporal correlation to spontaneous depolarizations associated with retinal waves. Together, these findings indicate that retinal waves induce oscillations in cAMP levels and PKA activity in RGCs.

\section{Materials and Methods}

Dissociated cultures. Dissociated neurons and glia from postnatal day 1 (P1) rat retinas were plated on coverslips, with a subset of cultures placed on superior colliculus cultures from littermates plated the day before as described previously (Colicos et al., 2004). Cells were plated on surfaces treated with $10 \mu \mathrm{g} / \mathrm{ml}$ poly-D-lysine (70 kDa; Sigma, St. Louis, MO) overnight, followed by $2 \mu \mathrm{g} / \mathrm{ml}$ laminin for $2 \mathrm{~h}$ at $37^{\circ} \mathrm{C}$. Retinal neurons were plated at a density of $2 \times 10^{4}$ cells $/ \mathrm{cm}^{2}$ in serum-free Neurobasal medium [serum free culture media (SFCM)] supplemented with B27 (Invitrogen, Carlsbad, CA), $0.6 \%$ glucose, $2 \mathrm{~mm}$ glutamine, $10 \mathrm{~mm}$ HEPES, $1 \mathrm{~mm}$ Na-pyruvate, $50 \mu \mathrm{g} / \mathrm{ml}$ penicillin G, $50 \mathrm{U} / \mathrm{ml}$ streptomycin, $2.5 \mu \mathrm{g} / \mathrm{ml}$ insulin, $6 \mu \mathrm{M}$ forskolin, $10 \mathrm{ng} / \mathrm{ml} \mathrm{CNTF}$, and $50 \mathrm{ng} / \mathrm{ml}$ BDNF. Forskolin was added to the medium to ensure survival of RGCs (Meyer-Franke et al., 1995). It is not known whether forskolin enhances cell survival by elevating baseline levels of activity or by influencing periodic activation of the cAMP/PKA pathway. To avoid complications with the interpretation of cAMP imaging experiments, cultures were removed from forskolin-containing media for $10 \mathrm{~h}$ before experiments. Control experiments indicated stable baselines of cAMP after this treatment.

Transfections. Transfection of dissociated neurons was performed by electroporation using an exponentially decaying pulse. Acutely isolated whole retinas from $\mathrm{P} 0-\mathrm{P} 2$ rats were mounted on filter paper precoated with $100 \mu \mathrm{g} / \mu \mathrm{l}$ poly-D-lysine and $50 \mu \mathrm{g} / \mu \mathrm{l}$ laminin. They were incubated in dissection solution (Colicos et al., 2004) containing plasmid DNA (concentration of $\sim 0.2 \mu \mathrm{g} / \mu \mathrm{l}$ ) for $10 \mathrm{~min}$, electroporated with two square wave pulses with an electric field strength of $6.75 \mathrm{~V} / \mathrm{mm}$, allowed to recover for $10 \mathrm{~min}$ in dissection solution, and cultured in SFCM-A (same media as above, except using Neurobasal-A). Overnight before imaging, electroporated retinas were transferred to SFCM-A without forskolin. For recordings, explants are placed ganglion cell layer up in a temperature-controlled chamber $\left(30^{\circ} \mathrm{C}\right.$; Warner Instruments, Hamden, CT) mounted on the stage of an upright microscope (Axioskop; Zeiss, Oberkochen, Germany) and perfused continuously with artificial CSF (ACSF) (in mM): $119 \mathrm{NaCl}, 2.5 \mathrm{KCl}, 1.3 \mathrm{MgCl}_{2}, 1.0 \mathrm{~K}_{2} \mathrm{HPO}_{4}, 2.5 \mathrm{CaCl}_{2}$, 26.2 $\mathrm{NaHCO}_{3}$, and 11 D-glucose (bubbled with $95 \% \mathrm{O}_{2} / 5 \% \mathrm{CO}_{2}$ ). Explant health was assayed using whole-cell recording to measure resting potentials, action potentials, and spontaneous depolarizations (supplemental Fig. 1, available at www.jneurosci.org as supplemental material). Imaging experiments without independent evidence of retinal waves in explants were discarded from analysis.

Imaging. Live imaging of fluorescence resonance energy transfer
(FRET) indicators was performed on an upright Zeiss Axioskop 2, using a $60 \times$ LUMPLFLW water immersion objective (Olympus Optical, Tokyo, Japan). Individual FRET channel detection was accomplished by using a dual-view image splitter with appropriate yellow and cyan channel filters. Images were captured on a PhotoMetrics (Huntington Beach, CA) CoolSnap HQ CCD camera and analyzed in MetaMorph version 6.3 (Universal Imaging Corporation, West Chester, PA). Background fluorescence was subtracted from both channels, and cyan fluorescent protein (CFP) bleed-through into the yellow fluorescent protein (YFP) channel was corrected $\left(F_{\mathrm{YFP}}=F_{\mathrm{FRET}}-0.51 \times F_{\mathrm{CFP}}\right)$ (Gordon et al., 1998; van Rheenen et al., 2004).

We conducted acceptor photobleaching experiments to measure FRET efficiency. FRET efficiency is defined as $E=1-\left(F_{\mathrm{DA}} / F_{\mathrm{D}}\right)$, where $F_{\mathrm{DA}}$ is the intensity of the donor (in our case, CFP) in the presence of the acceptor (YFP), and $F_{\mathrm{D}}$ is the intensity of the donor in the absence of the acceptor. In our experiments, YFP was only partially photobleached, so to compute $F_{\mathrm{D}}$, we used the equation $F_{\mathrm{D}}=\left(F-F_{\mathrm{DA}}\right) / x+F_{\mathrm{DA}}$, where $F$ is the CFP intensity after partial photobleaching of YFP, and $x=\left(F_{\mathrm{A} \text {-pre }}-\right.$ $\left.F_{\text {A-post }}\right) / F_{\mathrm{A} \text {-pre }}$, the fraction of the acceptor that is photobleached.

FRET ratios are calculated as $R=\left(F_{\mathrm{YFP}} / F_{\mathrm{CFP}}\right)$. The magnitude of drug or depolarization-induced change was represented by the change in FRET ratio. Changes in FRET ratio, $\Delta R$, were computed by subtracting the value of the FRET ratio averaged over five images before manipulation from the value of the FRET ratio averaged over five images around the maximum response within $40 \mathrm{~s}$ after the drug application or depolarization. We observed oscillations in baseline lasting one to three frames; therefore, an average of five frames (corresponding to $10 \mathrm{~s}$ ) gave an accurate measure of the baseline without decrementing the amplitude of the induced changes. Note, for the cAMP sensor or ICUE2, increases in cAMP levels lead to a decrease in FRET efficiency. For ease of comparison, ratio changes for all three indicators are represented as the absolute value of $\Delta R=\left(F_{\mathrm{YFP}} / F_{\mathrm{CFP}}\right)_{\text {control }}-\left(F_{\mathrm{YFP}} / F_{\mathrm{CFP}}\right)_{\text {manipulation. }}$ Live $\mathrm{Ca}^{2+}$ imaging was performed with the same equipment as for FRET imaging. Fura-2 AM loading was performed as described previously (Bansal et al., 2000).

All pharmacological agents were purchased from Sigma. External solution for dissociated neurons contained the following (in $\mathrm{mm}$ ): $5 \mathrm{KCl}$, $123 \mathrm{NaCl}, 3 \mathrm{CaCl}_{2}, 2 \mathrm{MgCl}_{2}, 10$ glucose, and 10 HEPES, pH adjusted with $\mathrm{NaOH}$ to 7.3. High-potassium solution was similar, with $100 \mathrm{~mm} \mathrm{KCl}$ substituted for an equivalent amount of $\mathrm{NaCl}$. Short applications of dopamine or high- $\mathrm{K}^{+}$external solution were delivered through a glass electrophysiology electrode $(\sim 2 \mu \mathrm{m}$ tip size, located $<20 \mu \mathrm{m}$ from transfected neuron) using a PV830 Pneumatic PicoPump at 2-10 psi, 50 ms pulse duration at $4 \mathrm{~Hz}$ to RGCs in retinal explants. The duration of dopamine and potassium applications was altered by increasing the number of pulses. For experiments with 0 external calcium, ACSF was modified to replace $2.5 \mathrm{mM} \mathrm{CaCl}_{2}$ with $2 \mathrm{~mm}$ EGTA and $1.7 \mathrm{~mm} \mathrm{MgCl}_{2}$.

Electrophysiology. Whole-cell voltage-clamp recordings from retinal explants were made using an Axopatch 200B amplifier and pClamp6 software (Molecular Devices, Palo Alto, CA). The intracellular solution consisted of the following (in $\mathrm{mM}$ ): 98.3 potassium-gluconate, $1.7 \mathrm{KCl}$, 0.6 EGTA, $5 \mathrm{MgCl}_{2}$, $2 \mathrm{Na}_{2}$-ATP, $0.3 \mathrm{GTP}$, and 40 HEPES, pH 7.25 with $\mathrm{KOH}$. The calculated $E_{\mathrm{Cl}}$ was $-60 \mathrm{mV}$ in ACSF. Whole-cell currentclamp recordings were used to compare spontaneous depolarizations recorded from explants and acutely isolated retinas (supplemental Fig. 1, available at www.jneurosci.org as supplemental material). The duration of spontaneous depolarization was defined as the time between membrane potential deviations from baseline that were more than two times the root mean square (which was $\sim 0.5 \mathrm{mV}$ at $5 \mathrm{kHz}$ sampling).

Cell-attached recordings were used to depolarize RGCs transfected with AKAR2.2. We were unable to use whole-cell current-clamp recordings because dialyzing the contents of the cell in whole-cell configuration led to significantly decreased sensitivity of the indicator. Gigaohm seals were obtained with a series resistance of $\sim 500 \mathrm{M} \Omega$. Depolarizing pulses of $150 \mathrm{pA}$, lasting $1-3 \mathrm{~s}$, caused local increases in membrane potential of $\sim 150 \mathrm{mV}$. 


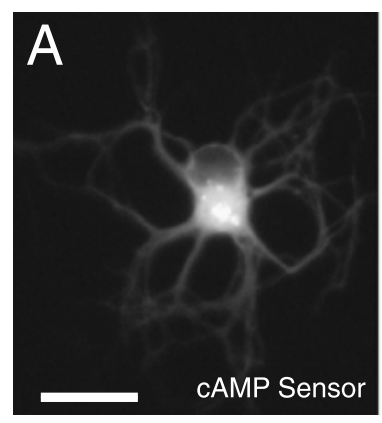

$\mathrm{D}$

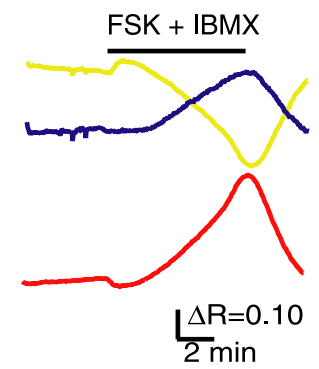

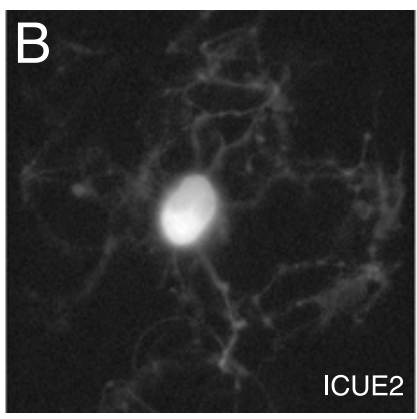

E

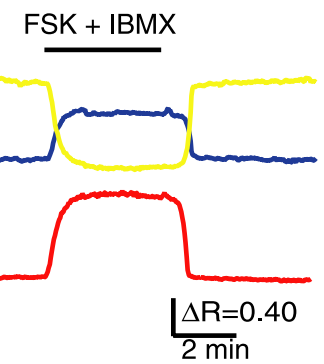

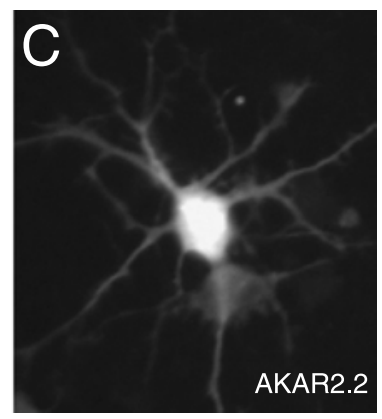

$\mathrm{F}$

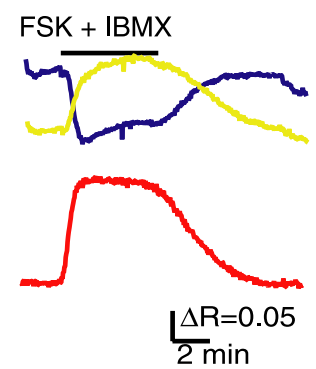

G

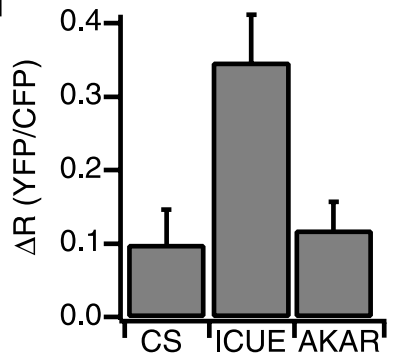

Figure 1. Expression of functional indicators in dissociated retinal neurons. $\boldsymbol{A}-\boldsymbol{C}$, Fluorescence images of cultured retinal neurons expressing the CAMP sensor $(\boldsymbol{A})$, ICUE2 (B), and AKAR2.2 ( $\boldsymbol{C}$ ). Retinal neurons were dissociated at $\mathrm{P} 0$ and imaged after $2 \mathrm{~d}$ in culture. Scale bar, $20 \mu \mathrm{m} . \boldsymbol{D}-\boldsymbol{F}$, Time course of $F_{\text {CFP }}$ (blue), $F_{\text {YFP }}$ (yellow) (imaged simultaneously), and the FRET ratio (red) of $F_{\text {YFP }} / F_{\text {CFP }}$ for the CS (D), ICUE2 (E), and AKAR2.2 (F) during application of both the adenylate cyclase activator forskolin (10 $\mu \mathrm{M}$ ) (FSK) and phosphodiesterase inhibitor IBMX (100 $\mu \mathrm{M})$. The bar represents time of drug applications. All ratios are corrected for CFP bleed-through into YFP channel and differential bleaching of the two fluorophores. Ratio traces for CS and ICUE2 are inverted to show increases in cAMP concentration as upward deflections. G, Summary of maximal FRET ratio $(\Delta R)$ changes in response to both forskolin and IBMX. $\Delta R$ was computed by subtracting the value of the FRET ratio averaged over five images before manipulation from the value of the FRET ratio averaged over five images around the maximum response.

\section{Results}

Expression of FRET indicators in dissociated retinal neurons

To monitor cAMP levels and PKA activity in living mammalian neurons, we used three different genetically encoded indicators dependent on FRET. The first, which we will refer to as the CS, uses an intermolecular FRET paradigm in which CFP is fused to a regulatory subunit of PKA and YFP is fused to the catalytic subunit of PKA (Zaccolo et al., 2002). An increase in cAMP concentration results in the binding of two cAMP molecules to the regulatory subunit of this fusion protein and a consequent release of the catalytic subunit. This results in a measurable decrease in FRET efficiency. The second indicator, ICUE2, consists of a fusion of CFP, a truncated form of Epacl including a cAMP binding site, and YFP [modified from ICUE1 (described by DiPilato et al., 2004)]. Binding of cAMP to the site on Epacl leads to a disruption of basal FRET interactions. The third indicator, AKAR2.2 (Zhang et al., 2005), is derived from AKAR2 (Zhang et al., 2001). Both AKAR2 and AKAR2.2 consist of a fusion of CFP, a phosphothreonine-binding domain (FHA1), a PKA consensus phosphorylation target, and the YFP variant citrine (Griesbeck et al., 2001). When PKA is activated, the catalytic subunit of PKA phosphorylates the target in AKAR2.2, causing a conformational change that increases FRET efficiency. AKAR2.2 differs from AKAR2 (Zhang et al., 2005) in that it includes A206K substitutions within both the CFP and YFP moieties to eliminate their tendency to dimerize (Zacharias et al., 2002). These mutations also greatly accelerate the phosphatase-mediated decrease in FRET efficiency that follows a PKA-induced increase in FRET efficiency (K. Kain, J. Zhang, L. Goldfinger, R. Tsien, M. Ginsberg, unpublished observations).

To determine whether the indicators were forming functional FRET pairs, a partial acceptor photobleach assay was performed for CS and AKAR2.2. For both indicators, $F_{\mathrm{CFP}}$ significantly in- creased after YFP was selectively photobleached using $515 \mathrm{~nm}$ light, indicating that, before stimulation, there was a baseline FRET interaction for both indicators. We measured FRET efficiencies of $0.11 \pm 0.09(n=9)$ and $0.18 \pm 0.06$ (average \pm SD; $n=10$ ) for CS and AKAR2.2, respectively (see Materials and Methods).

To characterize the physiological responses of CS, ICUE2, and AKAR2.2, the indicators were expressed in dissociated retinal neurons. Retinas from $\mathrm{P} 0-\mathrm{P} 1$ rats were dissociated and electroporated with plasmids encoding one of the three indicators. The neurons were plated and cultured in vitro for 2-4 d using conditions that promote survival of all classes of retinal neurons (Meyer-Franke et al., 1995; Goldberg et al., 2002a; Colicos et al., 2004). The indicators were strongly expressed throughout the cell (Fig. $1 A-C$ ). For comparisons across indicators, all ratio changes are represented as the absolute value of $\Delta R=\left(F_{\mathrm{YFP}} / F_{\mathrm{CFP}}\right)_{\text {control }}-$ $\left(F_{\mathrm{YFP}} / F_{\mathrm{CFP}}\right)_{\text {manipulation }}$.

We next determined the maximal response of the indicators to direct elevation of intracellular levels of cAMP. Neurons expressing CS, ICUE2, or AKAR2.2 exhibited large responses to bath application of both the adenylate cyclase activator forskolin (10 $\mu \mathrm{M})$ and the phosphodiesterase inhibitor IBMX (100 $\mu \mathrm{M})$, a manipulation that strongly stimulates the cAMP/PKA pathway (Fig. $1 D-G)\left(\Delta R_{\mathrm{CS}}=0.099 \pm 0.048, n=10 ; \Delta R_{\mathrm{ICUE}}=0.346 \pm 0.065\right.$, $\left.n=8 ; \Delta R_{\mathrm{AKAR}}=0.118 \pm 0.040, n=10\right)$. The response to forskolin alone was significantly smaller than the response to the combination of forskolin and IBMX for both AKAR2.2 $\left(\Delta R_{\mathrm{AKAR}}=0.044 \pm 0.014 ; n=8 ; p<0.01\right)$ and $\mathrm{CS}\left(\Delta R_{\mathrm{CS}}=\right.$ $0.012 \pm 0.005 ; n=8 ; p<0.01)$, demonstrating that the indicator response was correlated with the strength of activation of the cAMP/PKA pathway. We found no correlation between the starting ratio and the size of the FRET ratio change, indicating a lack of 
saturation of the indicator while in the basal state or with forskolin alone (data not shown).

\section{Evoked changes in cAMP levels and PKA activity in retinal explants}

To determine the effectiveness of indicators in monitoring activation of the cAMP/PKA pathway in the intact retina, we introduced the plasmids into neonatal rat retinal explants using electroporation (see Materials and Methods). After $20 \mathrm{~h}$, indicators were strongly expressed in RGCs, readily identified by the appearance of axons at the inner retinal surface (an example of an AKAR2.2-expressing RGC is shown in Fig. 2A).

First, we assessed the changes in PKA activity in response to brief application of forskolin $(50 \mu \mathrm{M})$. Short applications of forskolin caused a transient increase in the AKAR2.2 FRET ratio (Fig. $2 C)(\Delta R=$ $0.037 \pm 0.012 ; n=6$ neurons). The amplitude of the AKAR2.2 FRET ratio change did not change in calcium-free solutions (Fig. $2 C)(\Delta R=0.036 \pm 0.019 ; n=6$ neurons; $p=0.929)$. Hence, forskolin induced PKA activity in a calciumindependent manner.

Second, we investigated whether AKAR2. 2 could detect changes in PKA activity attributable to activation of G-protein-coupled receptors. Dopamine

$\mathrm{D}_{1}$ receptor immunolabeling is present throughout the inner plexiform layer in neonate rats (Koulen, 1999), and $D_{1}$ receptor, but not $\mathrm{D}_{2}$ receptor, $\mathrm{mRNA}$ is present in the ganglion cell layer of late prenatal rats (Schambra et al., 1994). $\mathrm{D}_{1}$-like receptors are positively coupled to adenylate cyclase; therefore, dopamine application in neonate rats is predicted to increase PKA activity. Indeed, brief application of dopamine $(150 \mu \mathrm{M})$ caused an increase in the AKAR2.2 FRET ratio in a majority of RGCs (Fig. $2 B$ ) ( $\Delta R=0.034 \pm 0.027 ; n=6$ of 8 neurons).

Third, we investigated whether the cAMP/PKA pathway in RGCs is activated by depolarization in retinal explants. RGCs express calcium-dependent adenylate cyclases (Abdel-Majid et al., 2002; Nicol et al., 2005), and therefore depolarization is likely to elevate cAMP/PKA levels (Ferguson and Storm, 2004). Short applications of external solution containing high concentrations of $\mathrm{KCl}(105 \mathrm{mM})$ to neurons expressing ICUE2 or AKAR2.2 induced changes in their FRET ratios (Fig. $2 C)\left(\Delta R_{\mathrm{ICUE}}=0.011 \pm\right.$ 0.003, $n=6$ neurons; $\Delta R_{\mathrm{AKAR}}=0.010 \pm 0.006, n=12$ neurons), approximately one-third the amplitude of those seen in response to forskolin or dopamine applications (Fig. 2C). Depolarizationinduced increases in AKAR2.2 FRET ratio were blocked in the presence of calcium-free external solution, implying that calcium influx is necessary for depolarization-induced activation of the cAMP/PKA pathway in RGCs. Depolarization-induced increases in the AKAR2.2 FRET ratio were significantly inhibited by the presence of $\mathrm{H} 89$ ( $N$-[2-( $p$-bromocinnamylamino)ethyl]-5isoquinolinesulfonamide $2 \mathrm{HCl})(25 \mu \mathrm{M})$, a specific PKA inhibitor (Zhang et al., 2001) (Fig. $2 D)\left(\Delta R_{\mathrm{H} 89} / \Delta R_{\text {control }}=0.25 \pm 0.10\right.$; $n=7 ; p<0.0001)$, confirming that AKAR2.2 was reporting increases in PKA activity.
Spontaneous oscillations in cAMP concentration and PKA activity are induced by and temporally correlated with retinal waves

We demonstrated that depolarization transiently activates the cAMP/PKA pathway. We next investigated the kinetics of the second-messenger pathway in response to spontaneous depolarizations associated with retinal waves. As described above, we introduced ICUE2 and AKAR2.2 plasmids into neonatal retinal explants using electroporation. Calcium imaging and whole-cell current-clamp recordings from individual RGCs in retinal explants revealed that RGCs exhibited correlated increases in intracellular calcium and periodic spontaneous depolarizations, consistent with the persistence of retinal waves after $48 \mathrm{~h}$ in culture (supplemental Fig. 1, available at www.jneurosci.org as supplemental material). However, there was a decrease in the duration of depolarization in explants compared with acutely isolated retina (explant, duration of $1.7 \pm 0.6 \mathrm{~s}, n=8$; acutely isolated retina, duration of $3.9 \pm 0.5 \mathrm{~s}, n=8$; $p<0.001)$. These shorter depolarizations were reflected in reduced amplitude calcium transients. Although $>95 \%$ of cells in the ganglion cell layer exhibited spontaneous calcium transients in both acutely isolated and explanted retinas, there was a stark difference in amplitude in most cells (supplemental Fig. 1C, available at www.jneurosci.org as supplemental material). Sixty percent of cells in explant retinas exhibited average fractional changes in fluorescence of $<4 \%$, whereas only $5 \%$ of cells in acutely isolated retinas had calcium transients that small.

Despite this decrease in depolarization and calcium influx, we detected spontaneous increases in FRET ratio in a subset of RGCs expressing either ICUE2 or AKAR2.2. $\left[\Delta R_{\mathrm{ICUE}}=0.015 \pm 0.011\right.$, 
A
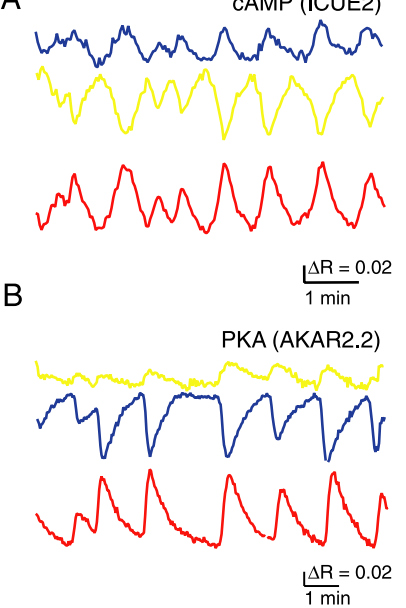

C
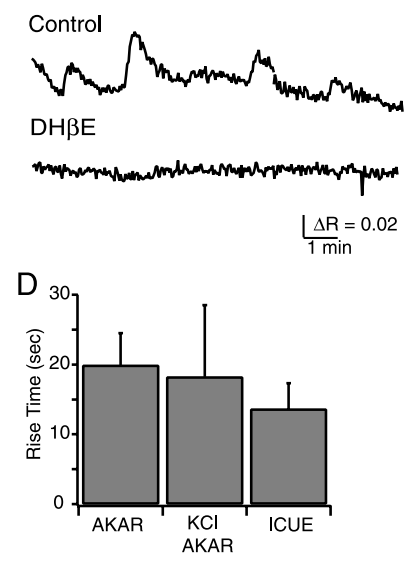

Figure 3. Spontaneous cAMP level and PKA activity changes induced by retinal waves. $\boldsymbol{A}$, Time course of $F_{\text {CPP }}$ (blue) and $F_{\text {YFP }}$ (yellow) recorded simultaneously and averaged over the cell body of a RGC expressing ICUE2. The ICUE2 FRET ratio is computed as $F_{\text {YFP }} / F_{\text {CFP }}$, inverted to show CAMP increases as upward deflections, corrected for CFP bleed-through into YFP channel, and corrected for differential bleaching of CFP and YFP. $B$, Time course of $F_{\text {CFP }}$ (blue) and $F_{\text {YFP }}$ (yellow) recorded simultaneously and averaged over the cell body of a RGC expressing AKAR2.2. The ratio is computed as $F_{\text {YFP }} / F_{\text {CFP }}$ corrected for CFP bleed-through into YFP channel, and corrected for differential bleaching. $C$, Blockade of retinal waves with the nicotinic acetylcholine receptor antagonist dihydro- $\beta$-erythroidine ( $\mathrm{DH} \beta \mathrm{E} ; 10-20 \mu \mathrm{m}$ ) blocked spontaneous PKA oscillations. $E$, Comparison of rise time of FRET ratio response to K-evoked and spontaneous events for ICUE2 and AKAR2.2.

$n=7$ of 31 RGCs (Fig. $3 A$ ); $\Delta R_{\text {AKAR }}=0.025 \pm 0.015, n=12$ of 96 RGCs (Fig. 3B)]. The magnitude of FRET ratio changes for both ICUE2 and AKAR2.2 were similar in amplitude and kinetics to those induced by acute application of potassium in RGCs (rise time measured trough to peak: ICUE2, $\tau_{\text {spontaneous }}=13.6 \pm 3.7 \mathrm{~s}$, $n=7$; AKAR2.2: $\tau_{\text {spontaneous }}=20.5 \pm 4.6 \mathrm{~s}, n=12 ; \tau_{\text {evoked }}=$ $18.2 \pm 10.3 \mathrm{~s}, n=14 ; p>0.5$ ) (Fig. $3 D$ ). These similarities suggest that retinal waves may lead to cAMP and PKA activity transients via depolarization.

Several observations indicate that spontaneous transients in PKA activity were induced by retinal waves. First, the spontaneous AKAR2.2 FRET ratio changes occurred with a periodicity similar to that of spontaneous depolarizations associated with retinal waves (peak-to-peak interval $2.05 \pm 0.94 \mathrm{~min} ; n=12$; $p=$ 0.173). Second, spontaneous AKAR2.2 FRET ratio changes were blocked by bath application of the nicotinic acetylcholine receptor antagonist dihydro- $\beta$-erythroidine $(10-20 \mu \mathrm{M})$, which reliably blocks retinal waves $(n=5)$ (Fig. $3 C)$. Third, in one instance in which we observed two transfected RGCs in the same field of view, we observed simultaneous AKAR2.2 FRET ratio changes (data not shown).

To determine whether the spontaneous PKA transients exhibit a consistent temporal relationship with retinal waves, we conducted simultaneous AKAR2.2 imaging and whole-cell voltage-clamp recordings from a nearby cell to detect the compound synaptic currents associated with retinal waves $(n=38$ waves from 4 cells) (Fig. $4 A$ ). We computed the "wave-triggered" AKAR2.2 FRET ratio change by averaging the segment of the FRET ratio for AKAR2.2 from $5 \mathrm{~s}$ preceding to $40 \mathrm{~s}$ after the compound PSC. We found that, on average, the AKAR2.2 FRET ratio increased after a barrage of synaptic current (Fig. $4 B$ ). For comparison, we computed the wave-triggered fura- 2 response using simultaneous whole-cell recording and calcium imaging. In contrast to the AKAR2.2 FRET ratio change, the calcium in-

A

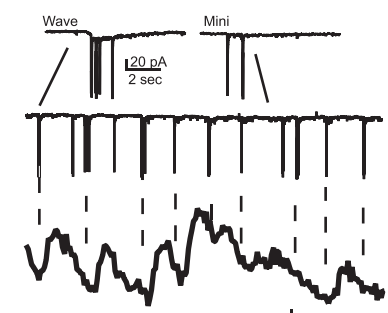

| $50 \mathrm{pA} \frac{\Delta \mathrm{R}=0.01}{1 \mathrm{~min}}$

C
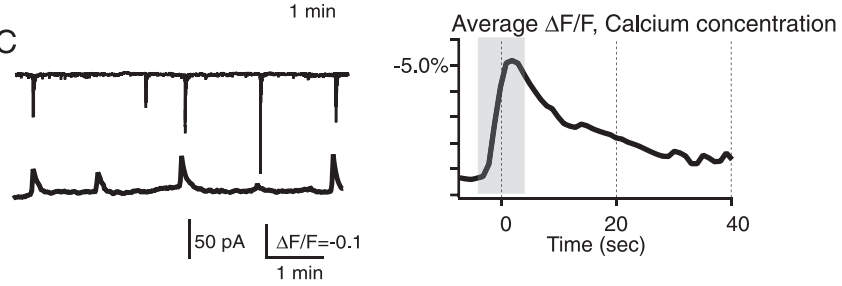

Figure 4. Spontaneous PKA transients are temporally correlated with retinal waves. $\boldsymbol{A}, \mathbf{S i}$ multaneous voltage clamp (top) and AKAR2.2 FRET imaging (bottom) from nearby retinal ganglion cells. Inset, Two events, a compound EPSC corresponding to a retinal wave (left) and single synaptic events (right). Wave-related events are distinguished by a slow inward current. $\boldsymbol{B}$, The wave-triggered average indicates that a barrage of synaptic currents associated with retinal waves is followed by an increase in PKA activity and preceded by a decrease in PKA activity from the previous transient. Gray bar indicates average length of compound PSCs. C, Simultaneous voltage-clamp recording (top) and fractional change in fluorescence of the calcium indicator fura-2, loaded via its AM ester (bottom), in nearby cells. $\boldsymbol{D}$, The wave-triggered average for $\mathrm{Ca}^{2+}$ transients indicate that barrages of synaptic currents are simultaneous with calcium increases. Gray bar indicates average length of compound PSCs.

crease in nearby cells occurred during the compound synaptic event (Fig. $4 C, D)(n=21$ waves from 5 cells). This is consistent with previous measurements that indicate that increases in intracellular calcium associated with retinal waves are driven by influx through voltage-gated channels during spontaneous depolarizations (Penn et al., 1998). Combined with the observation that blockade of retinal waves also blocks AKAR2.2 FRET ratio changes, the increase in FRET ratio after a retinal wave is consistent with the hypothesis that retinal waves induce PKA activity transients.

\section{Depolarization-induced activation of CAMP/PKA pathway requires 3-s-long depolarizations and calcium influx}

Why do we detect spontaneous PKA transients in only a subset of RGCs? In transfected RGCs that did not exhibit spontaneous oscillations in the FRET ratio, we measured substantial ratio changes in response to bath application of forskolin $(10 \mu \mathrm{M}$; $\Delta R=0.091 \pm 0.058 ; n=5$ of 5 neurons), demonstrating that the absence of oscillations is not attributable to a lack of functional AKAR2.2. In addition, we found that non-oscillating cells had detectable FRET ratio changes in response to strong depolarization either via short applications of high-potassium solutions or cell-attached recordings (Fig. 5). Based on these observations, we hypothesized that AKAR2.2 detected oscillations only in the RGCs that exhibited the strongest depolarizations during waves.

To quantify the magnitude of depolarization necessary to induce a PKA activity transient, we varied the duration of depolarization induced by short applications of a high-potassium solution (Fig. 5A, inset). Evoked FRET ratio changes and rise times are similar to those attributable to spontaneous PKA oscillations. We found that longer-duration depolarizations more reliably induced detectable changes in FRET ratio of AKAR2.2 $(n=11$ RGCs) (Fig. 5B). To verify that these changes in FRET ratio were 


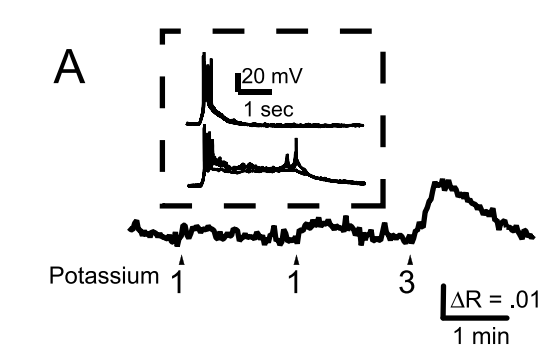

C
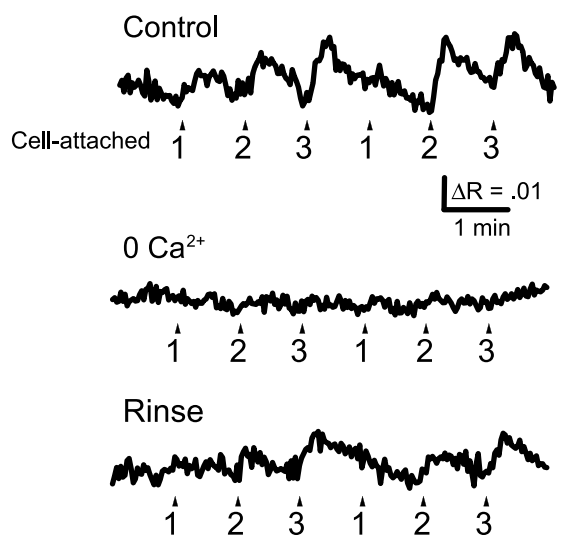

B

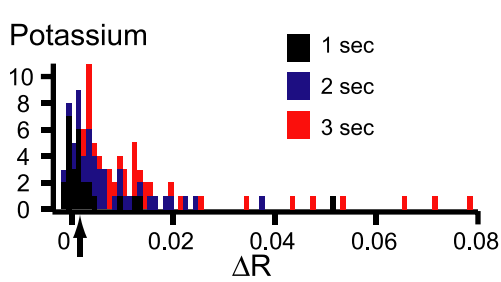

D

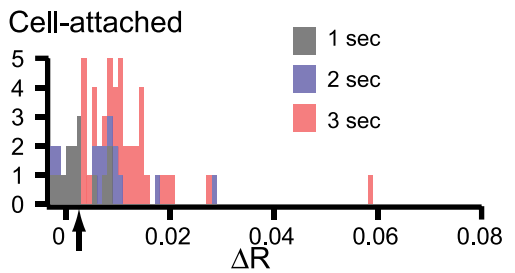

Cell-attached in $0 \mathrm{Ca} 2+$

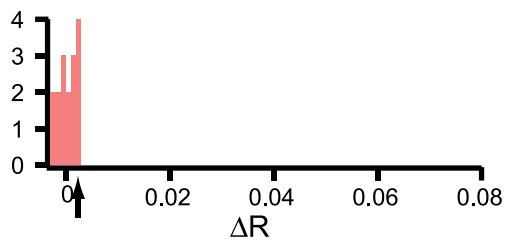

E

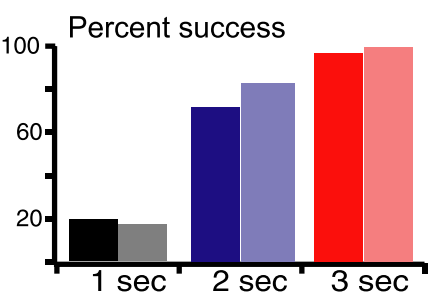

$\mathrm{F}$

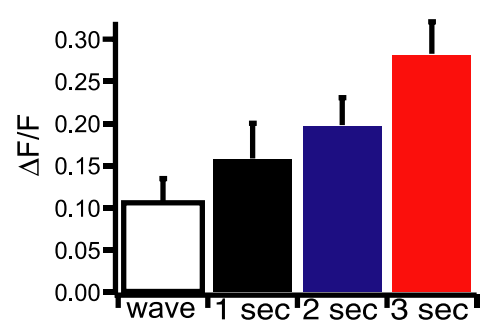

Figure 5. Three second depolarizations reliably evoked PKA activity transients in a calcium-dependent manner. $A$, Time course of AKAR2.2 FRET ratio $\left(F_{\text {YFP }} / F_{\text {CFP }}\right)$ in response to 1 or 3 s applications of solutions containing $105 \mathrm{~mm} \mathrm{~K}^{+}$. The arrows show the starting time for an application of high- $\mathrm{K}^{+}$solution. The numbers underlying the arrows indicate the duration of the potassium application. Inset, Current-clamp response of an $\mathrm{RGC}$ in response to $1 \mathrm{~s}$ (top) or $3 \mathrm{~s}$ (bottom) application of high- $\mathrm{K}^{+}$solution. $\boldsymbol{B}$, Histogram distribution of the change in FRET ratio ( $\Delta R$ ) in response to application of high- $\mathrm{K}^{+}$solution of varying duration (black for $1 \mathrm{~s}$ duration; blue for $2 \mathrm{~s}$ duration; red for 3 s duration). Arrow refers to threshold over which we could reliably distinguish responses from the noise. C, Time course of AKAR2.2 FRET ratio in response to depolarization of RGC neurons induced via a cell-attached pipette in the presence and absence of 0 Ca ${ }^{2+}$ ACSF. The arrows indicate the starting time for a given depolarization pulse. The numbers below the arrows indicate the duration of the depolarization pulses. $D$, Histogram distribution of the change in FRET ratio $(\Delta R$ ) to depolarization of RGC neurons induced via a cell-attached pipette of varying duration. Bottom, Histogram distribution of responses to $3 \mathrm{~s}$ depolarizations in the absence of extracellular calcium. Arrows refer to threshold over which we could reliably distinguish response from the noise. $\boldsymbol{E}$, The percentage of detectable depolarization induced changes in FRET ratio computed from histogram distributions in $\boldsymbol{B}$ and $\boldsymbol{D}$. For both potassium and electrophysiologically induced depolarizations, longer durations more reliably induced changes in FRET ratios. $\boldsymbol{F}$, Comparison of the fractional change of fluorescence of fura-2 AM induced by increases in intracellular calcium during retinal waves and calcium increases induced by applications of high- $K^{+}$solution of varying duration. All categories are significantly different from each other (paired Student's $t$ test, $p<0.0001$ ).

attributable to direct depolarization of RGCs, we reproduced these findings using a cell-attached patch electrode (Fig. 5C,D) ( $n=10$ RGCs). Using both stimulation methods, we found a significant increase in detectable AKAR2.2 FRET ratio changes in response to depolarizations $>2 \mathrm{~s}$ (Fig. $5 E$ ).

Based on these observations, we hypothesize that the low percentage of RGCs with spontaneous PKA activity transients in explants is attributable to the shorter spontaneous depolarization associated with retinal waves (supplemental Fig. 1, available at www.jneurosci.org as supplemental material). Hence, we would predict that, in acutely isolated retina, or more importantly in vivo, in which recent experiments report spiking bursts lasting 3-4 s (Hanganu et al., 2006), a larger percentage of RGCs exhibit PKA transients.

\section{Depolarization-induced PKA activity transients require $\mathrm{Ca}^{2+}$ influx}

Depolarization-induced activation of second-messenger pathways are proposed to function via calcium-dependent adenylate cyclases (Ferguson and Storm, 2004). Calcium imaging of RGCs loaded with fura-2 AM revealed that longer-duration depolarizations led to larger increases in intracellular calcium concentration (Fig. 5F). Hence, longer depolarizations increase the likelihood of PKA activity transients because they lead to a larger increase in intracellular calcium. In addition, we found that removal of calcium from ACSF abolished the depolarization-induced AKAR2.2
FRET ratio changes observed in RGCs (Fig. 5D) ( $n=17$ events from 4 RGCs and 4 retinas). Together, these findings indicate that PKA activity transients in RGCs are induced by depolarization in a calcium-dependent manner.

\section{Discussion}

We demonstrate that live indicators for cAMP levels and PKA activity can reliably measure activation of this second-messenger pathway in neurons in the intact circuitry of the developing retina. In addition, our findings for the first time link spontaneous neural activity in developing networks with temporal oscillations in kinase activity. This temporal coding of second-messenger pathway activation may be critical for the onset and timing of downstream developmental processes that are driven by spontaneous neural activity.

\section{Comparison of cAMP and PKA indicators}

We found that the genetically encoded indicators ICUE2 and AKAR2.2 reliably reported both evoked (Figs. 1, 2, 5) and spontaneous (Figs. 3, 4) changes in cAMP concentration and PKA activity in retinal neurons. These measurable changes in FRET efficiency occurred as responses to either direct [via stimulation of adenylate cyclase (Figs. $1 B, 2 A$ )] or indirect [via activation of a G-protein-coupled receptor or depolarization (Fig. $2 B-D$ )] activation of the cAMP/PKA pathway. One notable difference between the three indicators was the kinetics of the response to strong stimulation (Fig. 1D-F). The CS ratio change was much 
slower than that of ICUE2 and AKAR2.2. One possible explanation is that cAMP may activate PKA kinase activity before completely dissociating the regulatory and catalytic subunits (Yang et al., 1995; Nikolaev et al., 2004). For this reason, the CS FRET ratio changes occur only after PKA dissociates, whereas AKAR2.2 ratio changes occur faster. Similarly, ICUE2 FRET ratio changes will occur as cAMP concentration increases independent of PKA dissociation. In summary, we conclude that, in our system, ICUE2 and AKAR2.2 function as more sensitive reporters for monitoring brief activation of the cAMP/PKA pathway. These findings indicate that ICUE2 and AKAR2.2 can be used to observe activity of the cAMP/PKA pathway in vivo, for example in developing neocortex, in which strong calcium transients have been observed in newborn mice (Adelsberger et al., 2005). In addition, immobilized AKAR2.2 can be used to monitor subcellular compartments of PKA activity, such as those observed in growth cones (Terman and Kolodkin, 2004; Kain, Zhang, Goldfinger, Tsien, Ginsberg, unpublished observations).

\section{Potential function of periodic PKA activation in developing circuits}

AKAR2.2 FRET ratio changes were induced in nearly all RGCs with sufficient depolarization (Fig. 5). In our explants that had shorter spontaneous depolarizations than observed in acutely isolated retina (supplemental Fig. 1, available at www.jneurosci.org as supplemental material), retinal waves induce transient activation of the cAMP/ PKA pathway (Figs. 3, 4). This evidence suggests that most RGCs in vivo exhibit periodic activation of PKA.

There are several examples that demonstrate that temporal coding of second-messenger pathway activation is critical for influencing development (Berridge et al., 2000; Spitzer et al., 2004). For example, in developing Xenopus spinal neurons, the frequency of calcium transients is critical in establishing neurotransmitter phenotype (Gu and Spitzer, 1995; Borodinsky et al., 2004). Similarly, in the ventricular zone in neocortex (Owens et al., 2000) and retina (Pearson et al., 2002, 2004), calcium transients are important for proliferation of ventricular zone cells and the subsequent fate differentiation of postmitotic neurons. Later in development, after distinct neuronal classes are specified but before motoneurons innervate their targets, spontaneous activity in the developing spinal cord is frequency tuned to modulate the expression of proteins critical for pathfinding of motoneurons (Hanson and Landmesser, 2004). The frequency of calcium transients in growth cones has also been implicated in mediating interactions of the growth cone with the local environment (Henley and Poo, 2004). Periodic activation of isolated RGCs and the spontaneous activity in retinal explants profoundly increase the rate of axonal outgrowth in response to BDNF (Goldberg et al., $2002 \mathrm{~b}$ ). This enhancement of BDNF-induced outgrowth is blocked by adenylate cyclase inhibitors (Goldberg et al., 2002b), indicating that the endogenous pattern of retinal activity regulates axon outgrowth via this second-messenger cascade, possibly by increasing the surface expression of neurotrophin receptors (Meyer-Franke et al., 1998; Nagappan and Lu, 2005).

Whether periodic activation of RGCs is required for normal development of the visual system is not yet determined. Although it is well established that spontaneous retinal activity patterns are critical for the refinement of retinal projections to central targets, the precise role of correlated activity patterns is the subject of intense debate. In a knock-out mouse that has uncorrelated retinal ganglion cell firing instead of retinal waves (Bansal et al., 2000; McLaughlin et al., 2003), it has been shown recently that several features of both retinocollicular projections (McLaughlin et al., 2003; Chandrasekaran et al., 2005;
Mrsic-Flogel et al., 2005) and retinogeniculate projections (Rossi et al., 2001; Muir-Robinson et al., 2002; Grubb et al., 2003) were altered. In contrast, both pharmacological manipulations of activity patterns in ferret and a different knock-out mouse with altered retinal patterns develop normal refinement of retinal projections (Stellwagen and Shatz, 2002; Huberman et al., 2003; Torborg et al., 2005). By comparing the retinal firing patterns that drive or permit segregation with those that do not, we identified several candidate activity features that may be essential for this refinement process (Torborg et al., 2005).

The primary hypothesis that arose from these studies is that RGCs must fire high-frequency bursts with a slow periodicity for normal eye-specific refinement. Such an activity pattern would strongly activate both calcium-dependent and cAMP-dependent second-messenger cascades. However, a critical component of the argument that retinal waves are instructive for driving eye-specific segregation or retinotopic refinement is that the plasticity process that reads out this instructive signal must be able to distinguish firing from neighboring cells versus firing from distant cells. Therefore, the elongated PKA transients we observed in RGCs are not likely to provide an instructive signal for driving map refinement but rather may function in a cell-autonomous manner. However, the plasticity mechanisms that convert this firing pattern into long-term changes in cellular morphology remain to be determined.

Another potential role for PKA oscillations may be in the generation of retinal waves. Retinal waves are generated by a network of starburst amacrine cells (Zheng et al., 2004, 2006), and the frequency of retinal waves is determined by changes in cAMP levels in starburst amacrine cells (Zheng et al., 2006). Indeed, bath application of the PKA inhibitor Rp-cAMP blocks retinal waves (Stellwagen et al., 1999). The data presented here do not address the role of PKA activity in the generation of retinal waves because we recorded PKA activity exclusively in RGCs, which "read out" the activity of the starburst network (Feller et al., 1997; Zheng et al., 2006) but are not thought to participate in the generation of retinal waves. Whether cAMP levels or PKA activity spontaneously oscillate in developing starburst amacrine cells has yet to be determined.

Models of the complex interplay between calcium and cAMP levels demonstrate that changes in either the level or kinetics of intracellular cAMP or calcium concentrations can lead to dramatic changes in the emergent behavior of the intracellular network (Gorbunova and Spitzer, 2002). The implications of these models is that neural activity patterns can activate different spatiotemporal patterns of second-messenger networks, which in turn may be uniquely tuned to drive specific long-term cellular changes. For example, expression of BDNF, a neurotrophic factor critical for normal arborization of retinal ganglion cell dendrites and axons as well synapse formation (Cohen-Cory and Lom, 2004), is upregulated by increased intracellular calcium and cAMP concentration but not by stimulation of the cAMP pathway alone (Fukuchi et al., 2005). Similarly, surface expression of TrkB may also be regulated by neuronal activity and cAMP levels (Nagappan and Lu, 2005). This suggests that the pattern of intracellular calcium and cAMP oscillations caused by periodic depolarizations may be critical for determining mature morphology in the developing visual system.

In conclusion, we report data that support the hypothesis that retinal waves induce oscillations in CAMP concentration and PKA activity of RGCs. Visualization of second-messenger cascade kinetics is an important step in elucidating the link between activity and the mechanisms by which it influences development of neural circuits. 


\section{References}

Abdel-Majid RM, Tremblay F, Baldridge WH (2002) Localization of adenylyl cyclase proteins in the rodent retina. Brain Res Mol Brain Res 101:62-70.

Adelsberger H, Garaschuk O, Konnerth A (2005) Cortical calcium waves in resting newborn mice. Nat Neurosci 8:988-990.

Bansal A, Singer JH, Hwang B, Feller MB (2000) Mice lacking specific nAChR subunits exhibit dramatically altered spontaneous activity patterns and reveal a limited role for retinal waves in forming ON/OFF circuits in the inner retina. J Neurosci 20:7672-7681.

Berridge MJ, Lipp P, Bootman MD (2000) The versatility and universality of calcium signalling. Nat Rev Mol Cell Biol 1:11-21.

Borodinsky LN, Root CM, Cronin JA, Sann SB, Gu X, Spitzer NC (2004) Activity-dependent homeostatic specification of transmitter expression in embryonic neurons. Nature 429:523-530.

Chandrasekaran AR, Plas DT, Gonzalez E, Crair MC (2005) Evidence for an instructive role of retinal activity in retinotopic map refinement in the superior colliculus of the mouse. J Neurosci 25:6929-6938.

Cohen-Cory S, Lom B (2004) Neurotrophic regulation of retinal ganglion cell synaptic connectivity: from axons and dendrites to synapses. Int J Dev Biol 48:947-956.

Colicos MA, Firth SI, Bosze J, Goldstein J, Feller MB (2004) Emergence of realistic retinal networks in culture promoted by the superior colliculus. Dev Neurosci 26:406-416.

DiPilato LM, Cheng X, Zhang J (2004) Fluorescent indicators of cAMP and Epac activation reveal differential dynamics of cAMP signaling within discrete subcellular compartments. Proc Natl Acad Sci USA 101:16513-16518.

Dyachok O, Isakov Y, Sagetorp J, Tengholm A (2006) Oscillations of cyclic AMP in hormone-stimulated insulin-secreting beta-cells. Nature 439:349-352.

Feller MB, Butts DA, Aaron HL, Rokhsar DS, Shatz CJ (1997) Dynamic processes shape spatiotemporal properties of retinal waves. Neuron 19:293-306.

Ferguson GD, Storm DR (2004) Why calcium-stimulated adenylyl cyclases? Physiology (Bethesda) 19:271-276.

Fields RD, Lee PR, Cohen JE (2005) Temporal integration of intracellular $\mathrm{Ca}^{2+}$ signaling networks in regulating gene expression by action potentials. Cell Calcium 37:433-442.

Fukuchi M, Tabuchi A, Tsuda M (2005) Transcriptional regulation of neuronal genes and its effect on neural functions: cumulative mRNA expression of PACAP and BDNF genes controlled by calcium and cAMP signals in neurons. J Pharmacol Sci 98:212-218.

Goldberg JL, Klassen MP, Hua Y, Barres BA (2002a) Amacrine-signaled loss of intrinsic axon growth ability by retinal ganglion cells. Science 296:1860-1864.

Goldberg JL, Espinosa JS, Xu Y, Davidson N, Kovacs GT, Barres BA (2002b) Retinal ganglion cells do not extend axons by default: promotion by neurotrophic signaling and electrical activity. Neuron 33:689-702.

Gorbunova YV, Spitzer NC (2002) Dynamic interactions of cyclic AMP transients and spontaneous $\mathrm{Ca}^{2+}$ spikes. Nature 418:93-96.

Gordon GW, Berry G, Liang XH, Levine B, Herman B (1998) Quantitative fluorescence resonance energy transfer measurements using fluorescence microscopy. Biophys J 74:2702-2713.

Griesbeck O, Baird GS, Campbell RE, Zacharias DA, Tsien RY (2001) Reducing the environmental sensitivity of yellow fluorescent protein. Mechanism and applications. J Biol Chem 276:29188-29194.

Grubb MS, Thompson ID (2004) The influence of early experience on the development of sensory systems. Curr Opin Neurobiol 14:503-512.

Grubb MS, Rossi FM, Changeux JP, Thompson ID (2003) Abnormal functional organization in the dorsal lateral geniculate nucleus of mice lacking the beta 2 subunit of the nicotinic acetylcholine receptor. Neuron 40:1161-1172.

Gu X, Spitzer NC (1995) Distinct aspects of neuronal differentiation encoded by frequency of spontaneous $\mathrm{Ca}^{2+}$ transients. Nature 375:784-787.

Hanganu IL, Ben-Ari Y, Khazipov R (2006) Retinal waves trigger spindle bursts in the neonatal rat visual cortex. J Neurosci 26:6728-6736.

Hanson MG, Landmesser LT (2004) Normal patterns of spontaneous activity are required for correct motor axon guidance and the expression of specific guidance molecules. Neuron 43:687-701.

Henley J, Poo MM (2004) Guiding neuronal growth cones using $\mathrm{Ca}^{2+}$ signals. Trends Cell Biol 14:320-330.

Huberman AD, Wang GY, Liets LC, Collins OA, Chapman B, Chalupa LM
(2003) Eye-specific retinogeniculate segregation independent of normal neuronal activity. Science 300:994-998.

Koulen P (1999) Postnatal development of dopamine D1 receptor immunoreactivity in the rat retina. J Neurosci Res 56:397-404.

Lu HC, Butts DA, Kaeser PS, She WC, Janz R, Crair MC (2006) Role of efficient neurotransmitter release in barrel map development. J Neurosci 26:2692-2703.

McLaughlin T, Torborg CL, Feller MB, O’Leary DD (2003) Retinotopic map refinement requires spontaneous retinal waves during a brief critical period of development. Neuron 40:1147-1160.

Meister M, Wong RO, Baylor DA, Shatz CJ (1991) Synchronous bursts of action potentials in ganglion cells of the developing mammalian retina. Science 252:939-943.

Meyer-Franke A, Kaplan MR, Pfrieger FW, Barres BA (1995) Characterization of the signaling interactions that promote the survival and growth of developing retinal ganglion cells in culture. Neuron 15:805-819.

Meyer-Franke A, Wilkinson GA, Kruttgen A, Hu M, Munro E, Hanson Jr MG, Reichardt LF, Barres BA (1998) Depolarization and cAMP elevation rapidly recruit TrkB to the plasma membrane of CNS neurons. Neuron 21:681-693.

Mrsic-Flogel TD, Hofer SB, Creutzfeldt C, Cloez-Tayarani I, Changeux JP, Bonhoeffer T, Hubener M (2005) Altered map of visual space in the superior colliculus of mice lacking early retinal waves. J Neurosci 25:6921-6928.

Muir-Robinson G, Hwang BJ, Feller MB (2002) Retinogeniculate axons undergo eye-specific segregation in the absence of eye-specific layers. J Neurosci 22:5259-5264.

Nagappan G, Lu B (2005) Activity-dependent modulation of the BDNF receptor TrkB: mechanisms and implications. Trends Neurosci 28:464-471.

Nicol X, Muzerelle A, Bachy I, Ravary A, Gaspar P (2005) Spatiotemporal localization of the calcium-stimulated adenylate cyclases, AC1 and AC8, during mouse brain development. J Comp Neurol 486:281-294.

Nicol X, Muzerelle A, Rio JP, Metin C, Gaspar P (2006) Requirement of adenylate cyclase 1 for the ephrin-A5-dependent retraction of exuberant retinal axons. J Neurosci 26:862-872.

Nikolaev VO, Bunemann M, Hein L, Hannawacker A, Lohse MJ (2004) Novel single chain cAMP sensors for receptor-induced signal propagation. J Biol Chem 279:37215-37218.

Owens DF, Flint AC, Dammerman RS, Kriegstein AR (2000) Calcium dynamics of neocortical ventricular zone cells. Dev Neurosci 22:25-33.

Pearson R, Catsicas M, Becker D, Mobbs P (2002) Purinergic and muscarinic modulation of the cell cycle and calcium signaling in the chick retinal ventricular zone. J Neurosci 22:7569-7579.

Pearson RA, Catsicas M, Becker DL, Bayley P, Luneborg NL, Mobbs P (2004) $\mathrm{Ca}^{2+}$ signalling and gap junction coupling within and between pigment epithelium and neural retina in the developing chick. Eur J Neurosci 19:2435-2445.

Penn AA, Riquelme PA, Feller MB, Shatz CJ (1998) Competition in retinogeniculate patterning driven by spontaneous activity. Science 274:2108-2112.

Pham TA, Rubenstein JL, Silva AJ, Storm DR, Stryker MP (2001) The cre/creb pathway is transiently expressed in thalamic circuit development and contributes to refinement of retinogeniculate axons. Neuron 31:409-420.

Plas DT, Visel A, Gonzalez E, She WC, Crair MC (2004) Adenylate cyclase 1 dependent refinement of retinotopic maps in the mouse. Vision Res 44:3357-3364.

Ravary A, Muzerelle A, Herve D, Pascoli V, Ba-Charvet KN, Girault JA, Welker E, Gaspar P (2003) Adenylate cyclase 1 as a key actor in the refinement of retinal projection maps. J Neurosci 23:2228-2238.

Rossi FM, Pizzorusso T, Porciatti V, Marubio LM, Maffei L, Changeux JP (2001) Requirement of the nicotinic acetylcholine receptor beta 2 subunit for the anatomical and functional development of the visual system. Proc Natl Acad Sci USA 98:6453-6458.

Ruthazer ES, Cline HT (2004) Insights into activity-dependent map formation from the retinotectal system: a middle-of-the-brain perspective. J Neurobiol 59:134-146.

Schambra UB, Duncan GE, Breese GR, Fornaretto MG, Caron MG, Fremeau RT Jr (1994) Ontogeny of D1A and D2 dopamine receptor subtypes in rat brain using in situ hybridization and receptor binding. Neuroscience 62:65-85.

Spitzer NC, Root CM, Borodinsky LN (2004) Orchestrating neuronal dif- 
ferentiation: patterns of $\mathrm{Ca}^{2+}$ spikes specify transmitter choice. Trends Neurosci 27:415-421.

Stellwagen D, Shatz CJ (2002) An instructive role for retinal waves in the development of retinogeniculate connectivity. Neuron 33:357-367.

Stellwagen D, Shatz CJ, Feller MB (1999) Dynamics of retinal waves are controlled by cyclic AMP. Neuron 24:673-685.

Terman JR, Kolodkin AL (2004) Nervy links protein kinase a to plexinmediated semaphorin repulsion. Science 303:1204-1207.

Torborg CL, Feller MB (2005) Spontaneous patterned retinal activity and the refinement of retinal projections. Prog Neurobiol 76:213-235.

Torborg CL, Hansen KA, Feller MB (2005) High frequency, synchronized bursting drives eye-specific segregation of retinogeniculate projections. Nat Neurosci 8:72-78.

van Rheenen J, Langeslag M, Jalink K (2004) Correcting confocal acquisition to optimize imaging of fluorescence resonance energy transfer by sensitized emission. Biophys J 86:2517-2529.

West AE, Griffith EC, Greenberg ME (2002) Regulation of transcription factors by neuronal activity. Nat Rev Neurosci 3:921-931.

Wong RO, Meister M, Shatz CJ (1993) Transient period of correlated bursting activity during development of the mammalian retina. Neuron 11:923-938.

Yang S, Fletcher WH, Johnson DA (1995) Regulation of cAMP-dependent protein kinase: enzyme activation without dissociation. Biochemistry 34:6267-6271.

Zaccolo M, Pozzan T (2003) CAMP and $\mathrm{Ca}^{2+}$ interplay: a matter of oscillation patterns. Trends Neurosci 26:53-55.

Zaccolo M, Magalhaes P, Pozzan T (2002) Compartmentalisation of cAMP and $\mathrm{Ca}^{2+}$ signals. Curr Opin Cell Biol 14:160-166.

Zacharias DA, Violin JD, Newton AC, Tsien RY (2002) Partitioning of lipidmodified monomeric GFPs into membrane microdomains of live cells. Science 296:913-916.

Zhang J, Ma Y, Taylor SS, Tsien RY (2001) Genetically encoded reporters of protein kinase A activity reveal impact of substrate tethering. Proc Natl Acad Sci USA 98:14997-15002.

Zhang J, Hupfeld CJ, Taylor SS, Olefsky JM, Tsien RY (2005) Insulin disrupts beta-adrenergic signalling to protein kinase A in adipocytes. Nature 437:569-573.

Zheng J, Lee S, Zhou ZJ (2006) A transient network of intrinsically bursting starburst cells underlies the generation of retinal waves. Nat Neurosci 9:363-371.

Zheng JJ, Lee S, Zhou ZJ (2004) A developmental switch in the excitability and function of the starburst network in the mammalian retina. Neuron 44:851-864. 